INTERNATIONAL DESIGN CONFERENCE - DESIGN 2018

https://doi.org/10.21278/idc.2018.0220



\title{
KNOWLEDGE IN ENGINEERING DESIGN: A SYSTEMATIC LITERATURE REVIEW ON ARTIFACTS AND IT SYSTEMS
}

\author{
M. Preidel, W. M. Wang, K. Exner and R. Stark
}

\begin{abstract}
Intense collaboration within networks of stakeholders characterizes current engineering design processes. In these, engineers use IT systems to create artifacts, which manifest their knowledge allowing its circulation. Still, a research gap exists regarding the understanding of kinds, relations and interdependencies between IT systems, artifacts and knowledge types. This article addresses this gap by presenting results of a systematic literature review. The results contribute to close the mentioned gap, give insight on focusses of current research and identify further need for investigations.
\end{abstract}

Keywords: design knowledge, virtual engineering (VE), design tools, engineering design, design artifacts

\section{Introduction}

Engineering design processes (EDP) can be seen as iterative problem solving processes for ill-structured problems which are characterized by a lack of knowledge in all process phases (Ullman, 2010; Neumann, 2015). In order to develop innovative and high quality products, engineers have to combine their abilities and expertise and thus, exchange data, information and knowledge across disciplinary and organizational borders. However, the terms data, information and knowledge are not understood in a uniform way by different disciplines (Neumann, 2015). According to North (2011), data can be seen as a basic resource from which information and knowledge can be derived, whilst data per se is merely a collections of symbols and of little use without context. By adding a context for data interpretation, it gains a meaning and becomes information. Knowledge can then be created by linkage of information, e.g. for a specific purpose, to enable problem solving or decision-making. Consequently, knowledge represents a key asset in EDP. It is worth mentioning that the term knowledge itself also has multiple facets. It is not uniformly understood and can be categorized along multiple dimensions. (Lugger and Kraus, 2001) In this paper, knowledge is understood as the result of linking and processing of information through an individual or a conscious mind (Lugger and Kraus, 2001; Wiater, 2007; North, 2011; Lehner, 2012). Nevertheless, artefacts such as documents or 3D models, which as a result of such processes actually only carry or manifest the applied knowledge, are often regarded as knowledge themselves in engineering or technical context. This is also reflected by the ASHEN model for knowledge categorization provided by Snowden (2000). The model describes implicit and explicit knowledge as the two basic dimensions and introduces five categories of knowledge, i.e. Artifacts, Skills, Heurstics, Experience, Natural talent, which are distributed in the continuum between the two dimensions (Snowden, 2000). Following the descriptions of the EDP and data, information and knowledge above, this article considers the EDP as a process during which engineers use available sources of data and information and apply their own stock of knowledge to generate solutions for given 
problems. Activities in this regard, such as data/information retrieval, part modelling etc., are carried out by using IT systems, such as product data management (PDM) systems or CAD tools. Created and manipulated artifacts are used as inputs to represent the result of the activity. Through the circulation of these artifacts and their further development within the process, the maturity level of the envisioned product advances steadily. Still, the interrelations between the applied engineering knowledge, the artifacts, which manifest it and the IT systems used in the EDP are not sufficiently understood in engineering practice. This shortcoming has been stated frequently within several industrial projects. In order to enable the change from the traditional, time-driven development process to a novel knowledgedriven one, however, these aspects must be understood.

This article presents the results of a systematic literature review, which focused on scientific literature on engineering design and the according IT systems, artifacts and knowledge types that were described in scientific discourse. The aim is to evaluate the focus of current research regarding knowledge in engineering design and which interrelations between IT system, artifacts and knowledge types have already been addressed so far. In Sections 2 and 3, the general approach of the research and an overview of analyzed literature are given. In Section 4, the results are presented and discussed with regard to their interrelations by applying network analytical methods. In Section 5, the results of the literature review as well as the network analysis are concluded and future research potentials are pointed out.

\section{Research approach}

A first step to increase the understanding of knowledge flows is an understanding of kinds, relations and especially interrelations between IT systems, artifacts and knowledge types. Therefore, the following question guides the research of this article: Which kinds, relations and especially interrelations concerning IT systems, artifacts and knowledge can be derived from current scientific literature? This question is answered by using the systematic literature review approach described in Petersen et al. (2008). This methodology was chosen, because it is suitable to identify publication fora and outlines methods for systematic classification as well as relation extraction. An overview of the actual research of this article is outlined in Figure 1. Firstly, relevant research objectives were defined to form the review scope. These were engineering design, artifacts, IT systems and knowledge. Only articles linked to the research areas engineering and computer science were considered relevant, because the research area engineering deals with the engineering design process, whereas computer science deals with IT systems. After experimenting with different time coverages, filtering for articles between 2007 and 2017 was assumed to yield the best trade-off between historical relevant and up-to-date literature. The thesaurus Technik und Management (TEMA) was used to get related, broad and narrow terms for each keyword set. Web Of Science ${ }^{\mathrm{TM}}$ (WOS) was the selected literature database, as it was the most extensive database available to the authors. The citation indexes SCI-EXPANDED and CPCI-S were used. The definition of the review scope results in four sets of keywords that were used for two queries. Table 1 presents the search strings. The keyword set \#1 relates to IT systems, set \#2 to engineering design, set \#3 to artifacts and set \#4 to knowledge. Query \#1 was a combination of the keyword sets \#1, \#2 and \#3; query \#2 combined keyword sets \#1, \#3 and \#4. The idea of set \#1 was to search for well-known IT systems relevant to product development. It was assumed that articles mentioning common engineering IT systems will also address uncommon IT systems. Therefore, a broad overview about IT systems used in product development was expected. Set \#2 increased the relevance of all other sets. This was achieved by combining set \#2 with the other sets. Set \#2 contained common terms related to virtual product creation, but the term itself was excluded from the search string, as it would had narrowed down the scope too strongly. Finding a search string addressing artifacts was difficult, because the term artifact is not the only term used for something resulting from engineering activities and created by an engineering IT system. Additionally, the term artifact is used within different contexts (e.g. noise in photographs). Therefore, set \#3 was designed in a very general manner. The broadest term to think of an artifact would be some kind of result, but including result as search term did not contribute to a relevant search. The term document is closely related to the term artifact, because it represents a digital or physical result of an activity. The narrow and broad terms of TEMA thesaurus provided sufficient terms for creating the search string of set $\# 4$ centered around knowledge. 


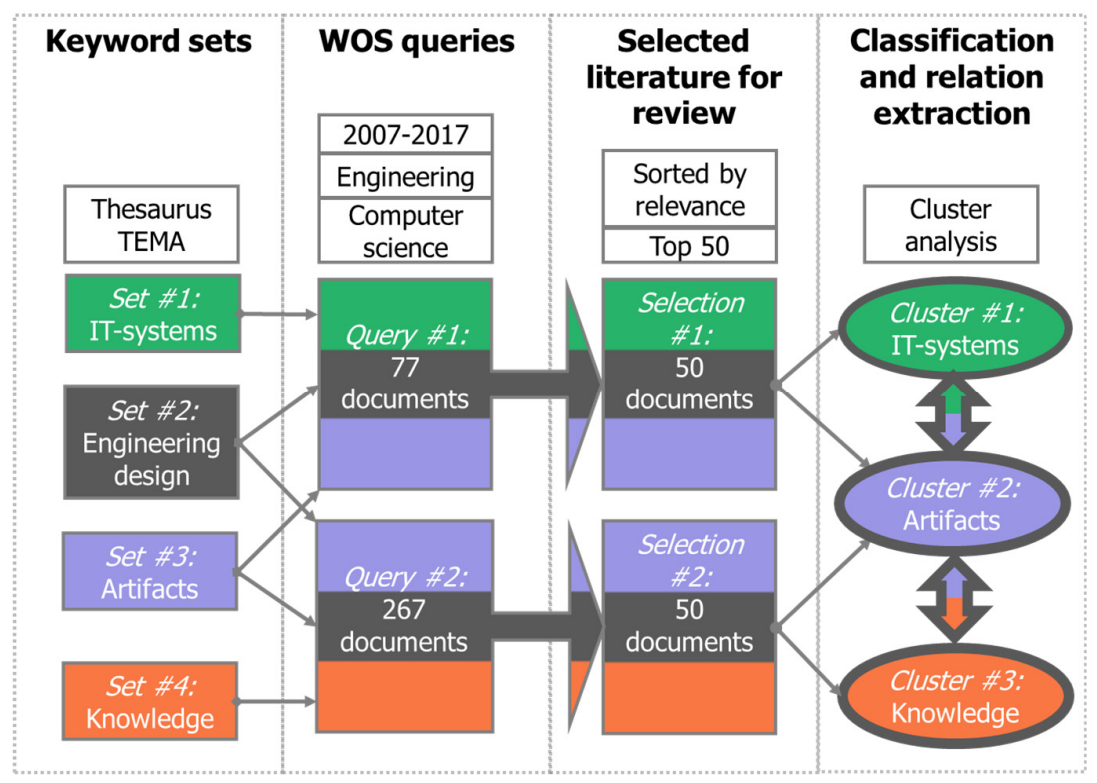

Figure 1. Research approach overview

Table 1. Search strings of sets used for literature acquisition

\begin{tabular}{|c|c|}
\hline $\begin{array}{l}\text { Set } \\
\text { ID }\end{array}$ & $\begin{array}{l}\text { Web of Science search string (TS: Topic, SU: Research area, OR/AND: logical operators, } \\
\text { \$: Wildcard for any character, "": Exact phrase) }\end{array}$ \\
\hline 1 & 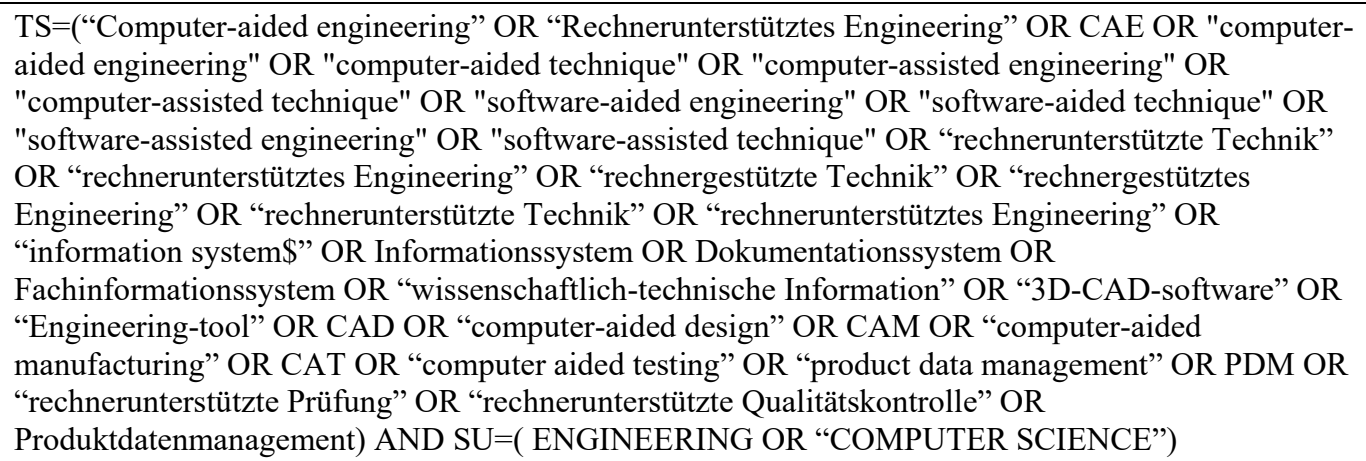 \\
\hline 2 & $\begin{array}{l}\text { TS=(“engineering design" OR Konstruktion OR "product development" OR Produktentwicklung OR } \\
\text { "product development process" OR Neuproduktentwicklung OR "design development" OR "model } \\
\text { designing" OR "model development" OR "Design-Entwicklung" OR Modellentwicklung OR } \\
\text { Neuentwicklung) ) AND SU=( ENGINEERING OR "COMPUTER SCIENCE") }\end{array}$ \\
\hline 3 & $\begin{array}{l}\text { TS }=(\text { Artifact OR Artefakt OR artefact OR documentation OR Dokumentation OR document OR } \\
\text { Dokument) AND SU=( ENGINEERING OR "COMPUTER SCIENCE") }\end{array}$ \\
\hline 4 & $\begin{array}{l}\text { TS=(Knowledge OR Wissen OR "Individual-related attributes" OR "Personenbezogenes Attribut" OR } \\
\text { "subjective properties" OR "subjektive Eigenschaften" OR "expert system" OR Expertise OR "know- } \\
\text { how" OR Expertensystem OR Expertenwissen OR "wissensbasiertes system" OR "Knowhow" OR } \\
\text { Sachkenntnis) AND SU=( ENGINEERING OR "COMPUTER SCIENCE") }\end{array}$ \\
\hline
\end{tabular}

Both queries yielded 344 documents in total. The results were sorted using the relevance algorithm provided by WOS. The 50 most relevant articles were selected for cluster analysis and relation extraction, because the least relevant 27 documents of selection \#1 started to be off-topic and both selections should be of equal size in order to get comparable results during cluster analysis. This resulted in 90 unique documents. The articles of both selections were read and analyzed in detail by the first author of this article. During analysis, the articles were tagged with keywords by found terms regarding IT systems, artifacts and knowledge as well as relations between the identified terms. If no full-text was available, the abstract was used for keyword tagging. Selection \#1 was analyzed regarding IT systems 
and artifacts, whereas the analysis of selection \#2 was focused on artifacts and knowledge. The classification scheme revealed synonymously used terms and hierarchies between terms. The data extraction and mapping process as well as the systematic map (in this article represented as networks) is subject to Section 4 .

\section{Literature overview}

The following section gives an in depth overview of the data that is analyzed in detail within Section 4. For increased readability, the combination of artifacts, IT systems and knowledge types is referred to as objects; connections between objects of any kind are referred to as links. Table 2 presents the selected articles for analysis. Due to limited page count, shortened bibliographic information are given in Table 2 , but full bibliographic information are available by contacting the corresponding author. Articles with only abstracts available are marked with shortened titles in italic. Articles contained in Selection \#1 and Selection \#2 are marked with an asterisk (*). The columns at the right hand side of the shortened title and year show the number of objects and links found within the related article in the same row. Table 2 is sorted descending by the sum of objects and links. The blue background color in the columns of year, objects and links represents the ranking of each value within its column. The darker the blue background, the higher the value compared to other articles. For example, in Chandrasegaran et al. (2013) the highest number of artifacts (25) was found among all articles. Therefore, the column Artifacts within the row with the shortened title Chandrasegaran, Ramani et al. 2013 - The Evolution, Challenges, And Future is colored in the darkest blue used for background coloring. A value of zero is marked with a white background, as it represents the lowest value possible. The numbers regarding objects and links were calculated as follows: The number of objects found within an article represents the number of unique objects found after elimination of synonyms closely related terms. For example, if the terms technical documentation, CAD file and CAD document occured within one article, the number of found artifacts was two for this article, because $C A D$ file and $C A D$ document are synonyms whereas technical documentation means a different artifact. Multiple mentions of the same object counted once. Links were counted in the same manner, while preserving the direction of the link. For example, consider the following case: An article mentions the links from CAD system to CAD file, CAD file to CAD system and design knowledge to technical drawings. The results are the following: Value 2 for the column Link artifact with IT system (CAD system to CAD file and CAD file to CAD system) and value 1 for the column Link artifact with knowledge (design knowledge to technical drawing). Overall, 28 IT systems, 126 artifacts and 78 knowledge types, 103 links between artifacts and IT systems as well as 61 links between artifacts and knowledge were identified.

Table 2. Literature overview with statistics regarding research objective

\begin{tabular}{l}
\hline \\
\end{tabular}


David and Rowe (2016) - What Does Plms Product Lifecycle

Tsao et al. (2013) - The Architecture For Solving

Toche et al. (2012) - Set-Based Prototyping With Digital Mock-Up

Stig (2013) - A Proposed Technology Platform Framework

Younis et al. (2014) - Applying Reverse Engineering And Its

Hahm et al. (2014) - A Personalized Query Expansion Approach

Leonardi and Bailey (2008) - Transformational Technologies And The

Creation

Pahng and Wall (2009) - Enhancing The Product Development Process

Pavkovic et al. (2013) - Facilitating Design Communication Through

Fantoni et al. (2013) - Automatic Extraction Of Function-Behaviour

Liu et al. (2016) - Intelligent Knowledge Recommending Approach *

Regli et al. (2011) - On The Long-Term Retention

Weber et al. (2009) - Searching Multiple Artifacts

Bretz et al. (2016) - A Concept For Managing Information

Bruun et al. (2014) - Interface Diagram

Veisz et al. (2012) - Computer-Aided Design Versus Sketching *

Desa and Munger (2013) - A Representation-Based Methodology For

Developing

Rockwell et al. (2010) - A Semantic Information Model

Bruun et al. (2015) - Plm system Support For Modular

Gopsill et al. (2015) - Supporting Engineering Design

Mayer et al. (2008) - Knowledge-Intensive Process Modelling In

Pernstal et al. (2012) - A Study Investigating Challenges

Groll and Heber (2016) - E/E-Product Data Management In Consideration

Hesse et al. (2016) - Decdoc: A Tool For Documenting

Liang et al. (2009) - Ombmdid: A Preliminary Attempt

Tweedale et al. (2016) - Advances In Intelligent Decision

Zhang et al. (2009) - Research On Collaborative Platform

Zdrahal et al. (2007) - Worlds And Transformations

Ansari-Ch et al. (2011) - Using Data Analysis For Discovering

Caldwell and Mocko (2009) - Product Data Management In Undergraduate

Chou (2014) - An Ideation Method For Generating

Nomaguchi et al. (2010) - Knowledge Management Framework*

Shan and He (2009) - Study Of Design And Analysis

Sirin et al. (2014) - Creating A Domain Ontology *

Consiglio et al. (2007) - Distributed Product Development

Florica and Draghici (2013) - Integrated Product Development Using Different

Jeon et al. (2016) - Automatic Cad Model Retrieval Based

Kohlhase et al. (2009) - Formal Management Of Cad/Cam Processes

Stelzer et al. (2012) - The Vr Session Manager

Udai and Sinha (2008) - Processing Magnetic Resonance Images

Gonnet et al. (2007) - A Model For Capturing

\begin{tabular}{|c|c|c|c|c|c|}
\hline 2016 & 9 & 0 & 7 & 0 & 7 \\
\hline 2013 & 19 & 0 & 3 & 0 & 0 \\
\hline 2012 & 9 & 0 & 3 & 0 & 9 \\
\hline 2013 & 1 & 12 & 0 & 7 & 0 \\
\hline 2014 & 16 & 3 & 0 & 1 & 0 \\
\hline 2014 & 4 & 4 & 0 & 11 & 0 \\
\hline 2008 & 7 & 0 & 4 & 0 & 8 \\
\hline 2009 & 13 & 1 & 0 & 5 & 0 \\
\hline 2013 & 7 & 9 & 0 & 3 & 0 \\
\hline 2013 & 2 & 12 & 0 & 2 & 0 \\
\hline 2016 & 5 & 4 & 1 & 5 & 1 \\
\hline 2011 & 10 & 0 & 2 & 0 & 4 \\
\hline 2009 & 6 & 0 & 7 & 0 & 3 \\
\hline 2016 & 6 & 0 & 7 & 0 & 2 \\
\hline 2014 & 10 & 0 & 2 & 0 & 3 \\
\hline 2012 & 2 & 1 & 3 & 4 & 5 \\
\hline 2013 & 11 & 2 & 0 & 1 & 0 \\
\hline 2010 & 7 & 4 & 0 & 3 & 0 \\
\hline 2015 & 7 & 0 & 1 & 0 & 4 \\
\hline 2015 & 9 & 2 & 0 & 1 & 0 \\
\hline 2008 & 6 & 6 & 0 & 0 & 0 \\
\hline 2012 & 4 & 5 & 0 & 3 & 0 \\
\hline 2016 & 5 & 0 & 3 & 0 & 3 \\
\hline 2016 & 6 & 3 & 0 & 2 & 0 \\
\hline 2009 & 3 & 7 & 0 & 1 & 0 \\
\hline 2016 & 3 & 8 & 0 & 0 & 0 \\
\hline 2009 & 10 & 1 & 0 & 0 & 0 \\
\hline 2007 & 7 & 3 & 0 & 0 & 0 \\
\hline 2011 & 7 & 1 & 0 & 0 & 0 \\
\hline 2009 & 2 & 0 & 2 & 0 & 4 \\
\hline 2014 & 2 & 5 & 0 & 1 & 0 \\
\hline 2010 & 4 & 1 & 1 & 1 & 1 \\
\hline 2009 & 6 & 0 & 1 & 0 & 1 \\
\hline 2014 & 2 & 2 & 2 & 1 & 1 \\
\hline 2007 & 6 & 1 & 0 & 0 & 0 \\
\hline 2013 & 4 & 0 & 2 & 0 & 1 \\
\hline 2016 & 5 & 0 & 1 & 0 & 1 \\
\hline 2009 & 5 & 0 & 1 & 0 & 1 \\
\hline 2012 & 5 & 0 & 2 & 0 & 0 \\
\hline 2008 & 3 & 0 & 1 & 0 & 3 \\
\hline 2007 & 5 & 1 & 0 & 0 & 0 \\
\hline
\end{tabular}


Zhang et al. (2013) - A Semantic Representation Model

Eddy et al. (2012) - Toward Integration Of A Semantic

Puodziuniene (2012) - Review Of Contemporary Cad systems

Restrepo (2007) - Assessing Relevance

Su et al. (2015) - Engineering Design Management And Service

Vermaas (2013) - The Coexistence Of Engineering Meanings

Erdim and Ilies (2007) - Detecting And Quantifying Envelope Singularities

Gonzalez et al. (2007) - Fostering Knowledge Mode

Iida et al. (2011) - Document Logic

Becker et al. (2009) - Developing Maturity Models

Crowder et al. (2009) - Knowledge-Based Repository To Support

Ericson et al. (2007) - On The Way To Knowledge

Flanagan et al. (2007) - Externalizing Tacit Overview Knowledge

Holzinger et al. (2008) - Typical Problems With Developing Mobile

Lundin et al. (2010) - Knowledge Retention And Reuse *

Schleich et al. (2016) - Skin Model Shapes

Xie et al. (2014) - Time Series Analysis Method

Yamamoto et al. (2010) - Thesaurus For Natural-Language-Based

Aurisicchio et al. (2008) - How To Evaluate Reading *

Hady and Wozny (2011) - Modularization Within The Framework

Hu et al. (2008) - An Xml-Based Implementation Of Manufacturing

Lejon et al. (2016) - Integrated Capture And Representation

Li and Ramani (2007) - Ontology-Based Design Information Extraction

Pan (2016) - Improving Maritime Technology

Rockwell et al. (2009) - A Web-Based Environment For

Ruocco et al. (2010) - Sketching In Design

Rytsareva et al. (2012) - Evaluating Socio-Technical Coordination In Open

Seminsky and Wessely (2008) - Approaches Of Layout Design Synthesis

Spiess and Anderl (2010) - Application Of Business Rules

Sui et al. (2015) - systematic Digitized Treatment Of Engineering

Vasiljevic et al. (2016) - Invention Reasoning Scheme Based

Boughzala and Vreede (2011) - A First Application

Cline (2009) - Organizational Barriers To The Implementation

Dahanayake and Thalheim (2011) - Enriching Conceptual Modelling

Practices

Kitamura and Mizoguchi (2010) - Some Ontological Distinctions Of Function

Pullan et al. (2010) - Application Of Concurrent Engineering

Rocha et al. (2010) - A Document-Oriented Web-Based Application

Scott and Perry (2012) - The Enactment Of Risk Categories

Sen et al. (2010) - Evaluation Of The Functional Basis

Fitterer (2010) - Information Model-Based Configuration Of Situational

Mckay et al. (2010) - An Exploration Of The Concept

Nakamura et al. (2012) - Reverse Engineering Tool Considering

Pollock and Hyysalo (2014) - The Business Of Being

\begin{tabular}{|c|c|c|c|c|}
\hline 2013 & 4 & 0 & 1 & 0 \\
\hline 2012 & 3 & 1 & 0 & 1 \\
\hline 2012 & 0 & 0 & 5 & 0 \\
\hline 2007 & 1 & 0 & 2 & 0 \\
\hline 2015 & 4 & 0 & 1 & 0 \\
\hline 2013 & 3 & 2 & 0 & 0 \\
\hline 2007 & 1 & 0 & 2 & 0 \\
\hline 2007 & 1 & 0 & 2 & 0 \\
\hline 2011 & 2 & 0 & 1 & 0 \\
\hline 2009 & 1 & 0 & 1 & 0 \\
\hline 2009 & 2 & 1 & 0 & 0 \\
\hline 2007 & 2 & 1 & 0 & 0 \\
\hline 2007 & 1 & 2 & 0 & 0 \\
\hline 2008 & 1 & 0 & 1 & 0 \\
\hline 2010 & 1 & 1 & . & 0 \\
\hline 2016 & 1 & 0 & 1 & 0 \\
\hline 2014 & 1 & 0 & 1 & 0 \\
\hline 2010 & 3 & 0 & 0 & 0 \\
\hline 2008 & 2 & 0 & 0 & 0 \\
\hline 2011 & 2 & 0 & 0 & 0 \\
\hline 2008 & 1 & 1 & 0 & 0 \\
\hline 2016 & 0 & 0 & 1 & 0 \\
\hline 2007 & 2 & 0 & 0 & 0 \\
\hline 2016 & 1 & 1 & 0 & 0 \\
\hline 2009 & 1 & 1 & 0 & 0 \\
\hline 2010 & 1 & 0 & 1 & 0 \\
\hline 2012 & 1 & 1 & 0 & 0 \\
\hline 2008 & 1 & 0 & 1 & 0 \\
\hline 2010 & 2 & 0 & 0 & 0 \\
\hline 2015 & 1 & 0 & 1 & 0 \\
\hline 2016 & 1 & 1 & 0 & 0 \\
\hline 2011 & 1 & 0 & 0 & 0 \\
\hline 2009 & 0 & 1 & 0 & 0 \\
\hline 2011 & 1 & 0 & 0 & 0 \\
\hline 2010 & 1 & 0 & 0 & 0 \\
\hline 2010 & 1 & 0 & 0 & 0 \\
\hline 2010 & 0 & 1 & 0 & 0 \\
\hline 2012 & 0 & 0 & 1 & 0 \\
\hline 2010 & 1 & 0 & 0 & 0 \\
\hline 2010 & 0 & 0 & 0 & 0 \\
\hline 2010 & 0 & 0 & 0 & 0 \\
\hline 2012 & 0 & 0 & 0 & 0 \\
\hline 2014 & 0 & 0 & 0 & 0 \\
\hline
\end{tabular}




\section{Results and discussion}

This section presents the results of the literature analysis. The results are discussed directly within the following sections. In Section 4.1 a one-directional overview about links from IT systems to artifacts and from artifacts to knowledge is given and analyzed. The Sections 4.2, 4.3 and 4.4 show bi-directional graph visualizations created with Gephi 0.9.2 (https://gephi.org/). All graphs use the following colorcoding: Purple stands for artifacts, green for IT systems and orange for knowledge types.

Only objects linked to other objects are visualized. In summary, eleven IT systems (38\%), 47 knowledge types $(60 \%)$ and 81 artifacts $(64 \%)$ were not linked to any other objects. From all objects, IT systems represented the smallest category, but they had the highest linking degree. The linking degree was defined as total number of outgoing or incoming links of any type. Compared to IT systems, there were four times more knowledge types and eight times more artifacts where no links to other objects could be extracted from literature. Overall, the number of unlinked objects was caused by the great variety of terms present in literature for knowledge types and especially artifacts. Regarding artifacts, a broad variety of terms was identified, that was presumably caused by different methodologies used in engineering design, different terminology of domains emphasizing specific aspects about an artifact and the circumstance, that different IT systems create different kinds of artifacts. Regarding knowledge types, many different levels and aspects of knowledge were identified: The same type of knowledge e.g. knowledge of an individual about best practices in product design - can be described as domain, design, implicit, product, process, common sense, product development, physical behavior and detailed solution knowledge.

\subsection{One-directional overview}

Figure 2 visualizes directed links between IT systems and artifacts with IT systems being the source of the link. The same applies to links between artifacts and knowledge with artifacts being the source of the link. This leads to complete linking chains from IT systems to knowledge with artifacts as connecting medium. Bold edges highlight these complete linking chains.

PDM/PLM systems brought up the greatest variety of artifacts (9), because PDM/PLM systems are built to be the engineering backbone managing all documents relevant for engineering. Hence, PDM/PLM systems traditionally are not an authoring system creating these artifacts, but nowadays PDM/PLM systems converge to a holistic engineering environment integrating various authoring functions like CAD modelling. 9 out of $13(69 \%)$ IT systems were the source of a single artifact. By far the most links (7) from IT systems to artifacts converged in unspecified documents. This means that scientific literature on IT systems was more focused on the systems themselves, rather than the resulting artifacts: The artifacts were often called documents without going deeper into details. Technical documentation (6), product model (4), unspecified documents (3) and CAD files (3) were the only artifacts with at least three outgoing (from artifact to knowledge) links to knowledge types. Knowledge stored in technical documentation spanned the product development phase from start to finish. It started with knowledge about requirements, continues to functional and product structure and finally stored knowledge about detailed solutions and the final product. Additionally, unspecified knowledge was stored.

To conclude, technical documentation stored various knowledge types related to all phases of EDP. The product model focused on daily business knowledge of a design engineer. It provided knowledge of two types: Process related and product related. The process related knowledge related to past design decisions, next steps and product development methodology in general. Product related knowledge was about interrelations to related product parts (e.g. available geometrical space). Literature described knowledge coming from unspecified documents as unspecified, domain or design knowledge. The same applies for CAD files: Knowledge provided by CAD files was described as unspecified, design knowledge or knowledge regarding potential problems. Obvious links - like product model to product knowledge - were not mentioned in literature and therefore not modelled during literature review. It was assumed, that these kind of obvious links awere not explicitly mentioned in literature, because they were well known and therefore uninteresting for research. Overall, 44 complete linking chains were found. A linking chain represents a unique path from IT system to knowledge (e.g. PDM/PLM system to CAD file and design knowledge). When following the bold links representing the complete linking chains, it 
was noticeable, that unspecified documents contributed to nearly $48 \%$ of the total amount of complete linking chains. The point that seven IT systems provided unspecified documents mainly caused this finding. Therefore, there is a need for further research on artifacts created by IT systems. Furthermore, 34 broken chains were found. An artifact not linked to at least one IT system or knowledge type characterizes a broken chain (e.g. NC code linked to CAD system but not linked to any knowledge). Missing links from IT systems to artifacts caused nearly $59 \%$ of broken chains, whereas missing links from artifacts to knowledge caused more than $41 \%$ of broken chains. While there were more linking chains broken due to missing links from artifacts to IT systems, the difference was not that high to conclude, that more research is needed to find links from IT systems to artifacts rather than from artifacts to knowledge. Therefore, further research is important in both directions.

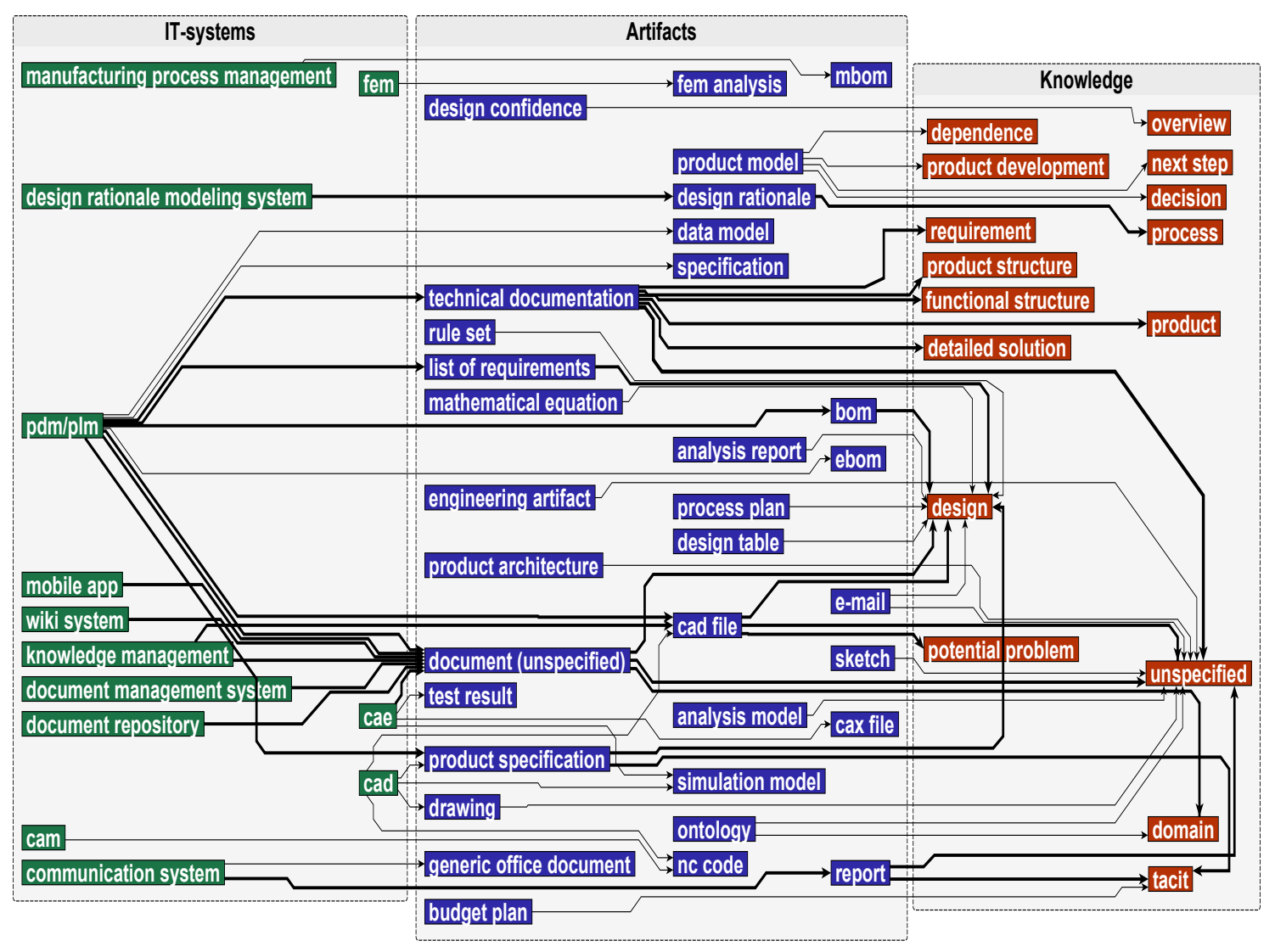

Figure 2. Complete and incomplete linking chains from IT system to knowledge

\subsection{Bi-directional network of IT systems and artifacts}

Figure 3 shows the biggest bi-directional network that resulted from combination of found IT systems and artifacts as well as the corresponding links between them. Biggest network is the term used for describing the network with the greatest number of connected nodes. The node size and edge thickness represent the number of articles mentioning the object (node) or link (edge). The edge color represents the source of a link (green: IT system, purple: artifact).

The top five artifacts by article count (values in parentheses) were the following: CAD files (35), unspecified documents (25), ontologies (21), BOMs (13) and technical documentations (12). 21 articles mentioned ontologies, but ontologies were not linked to nodes in the biggest network of IT systems and artifacts. This was caused by the finding that there were no links found between ontologies and any IT system (compare with Figure 2). The top five artifacts by linking degree were the following: Unspecified documents (21), ontologies (16), technical documentations (15), CAD files (14) and product specifications (6). It was noticeable, that the gap between place four (CAD files) and five (product 
specifications) was greater than $233 \%$. Moreover, place three (ontologies) was not linked to the biggest network - due to the reasons explained within the previous paragraph. Regarding linking degree, most relevant links between artifacts and IT systems were unspecified documents, technical documentations and CAD files.

The top five IT systems by article count were: CAD (24), PDM/PLM (15), CAE (7), document repository (6) and communication system (6). The top five IT systems by linking degree were PDM/PLM (18), CAE (8), CAD (6), knowledge management (4) and communication system (3). It was significant, that the linking degree of PDM/PLM was high compared to other IT systems. Nevertheless, a higher linking degree between PDM/PLM and CAD files was expected. Maybe this link is too common to be explicitly mentioned in scientific literature. The network around communication systems is not part of the biggest network shown in Figure 3. As shown in Figure 2, communication systems were the source of reports and generic office documents (like Microsoft Word or Microsoft Excel), providing unspecified and tacit knowledge. This revealed a starting point for further research: If generic office documents would be linked to unspecified documents, the network around communication systems would be connected to the biggest network. This new linking level between objects of the same type will contribute to increased understanding of knowledge flows.

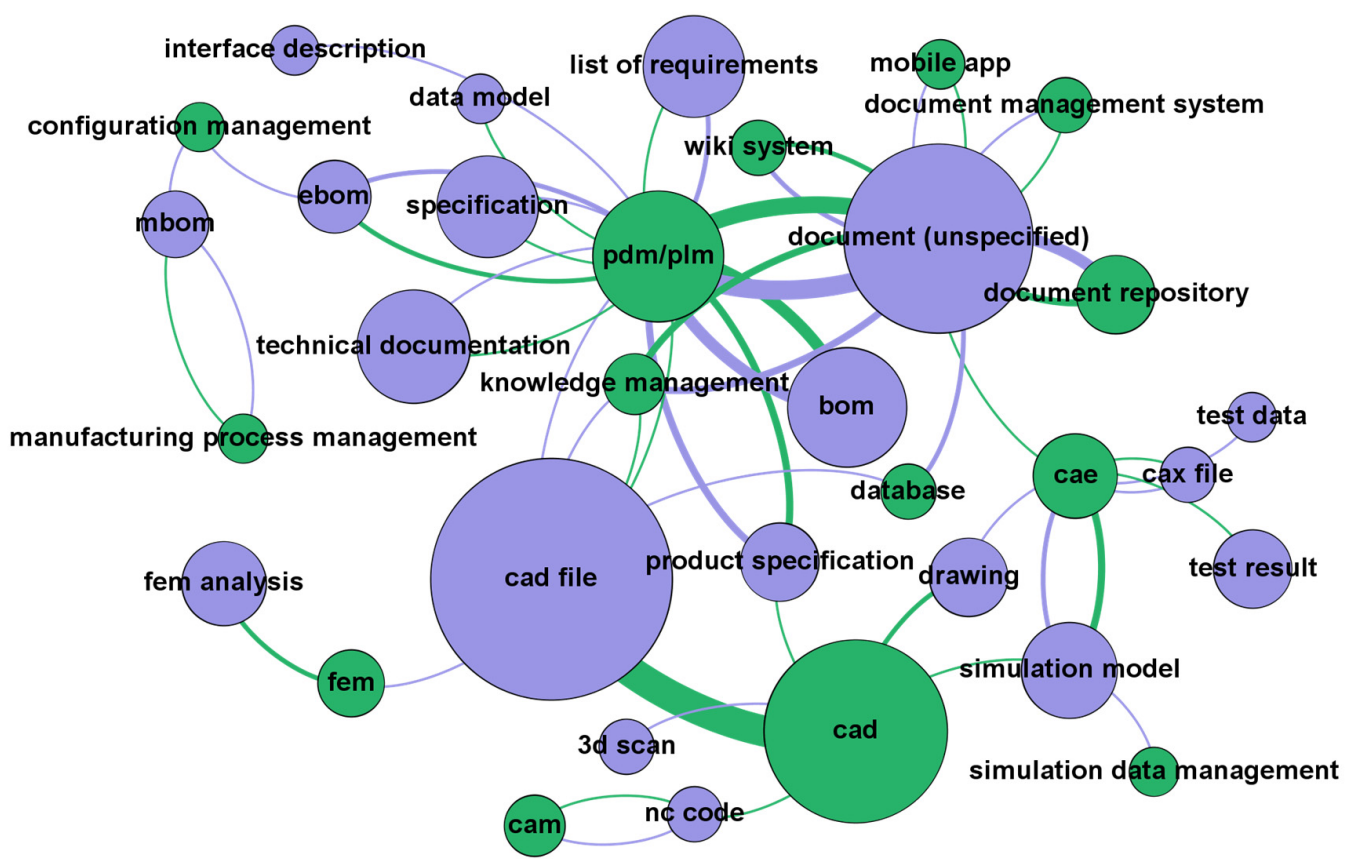

Figure 3. Biggest network of artifacts (green) and IT systems (purple)

By analyzing chains in Figure 3, the following aspects were noticeable: A linking chain well known from practice was found. It started with $\mathrm{BOM}$ and $\mathrm{BOM}$ (engineering $\mathrm{BOM}$ ) linked to PDM/PLM and continued from eBOM to configuration management to $\mathrm{mBOM}$ (manufacturing $\mathrm{BOM}$ ) and manufacturing process management. This is a good indicator that the research approach of this article is valid by comparing results found with well-known state of the art. Another aspect was the following: CAE systems were connected to CAD systems by simulation models and drawings. Whereas the link through simulation models was expected, Leonardi and Bailey (2008) mentioned the process of using drawings for computations in CAE systems. This was a hint that it is still relevant to do research regarding drawings, because drawings are still used in practice and not fully replaced by $3 \mathrm{D}$ models.

\subsection{Bi-directional network of artifacts and knowledge}

Figure 4 shows the biggest bi-directional network that resulted by combining identified knowledge and artifacts with the corresponding links between them. The node size and edge thickness represent the 
number of articles where the corresponding objects (nodes) and links (edges) were found. The edge color represents the source of a link (orange: knowledge, purple: artifact).

The top five artifacts by article count (values in parentheses) were the following: CAD files (35), unspecified documents (25), ontologies (21), BOMs (13) and technical documentations (12). The top five artifacts by linking degree were the following: Unspecified documents (21), ontologies (16), technical documentations (15), CAD files (14) and product specifications (6). Surprisingly, CAD files were mentioned the most with great distance to second place by article count (unspecified documents), but were at place four regarding the linking degree. This means that there was much research involving CAD files, but links to knowledge or IT systems for CAD files were not analyzed in detail by reviewed literature. The top five knowledge types by article count were the following: unspecified (14), design (14), domain (11), implicit (10) and historical (7) knowledge. The top five artifacts were mentioned $189 \%$ more often than the top five knowledge types by article count (56 vs. 106). There were $162 \%$ more artifacts than knowledge types in total. Therefore, research on artifacts concentrated significantly more on the top five artifacts than it was the case regarding knowledge types.

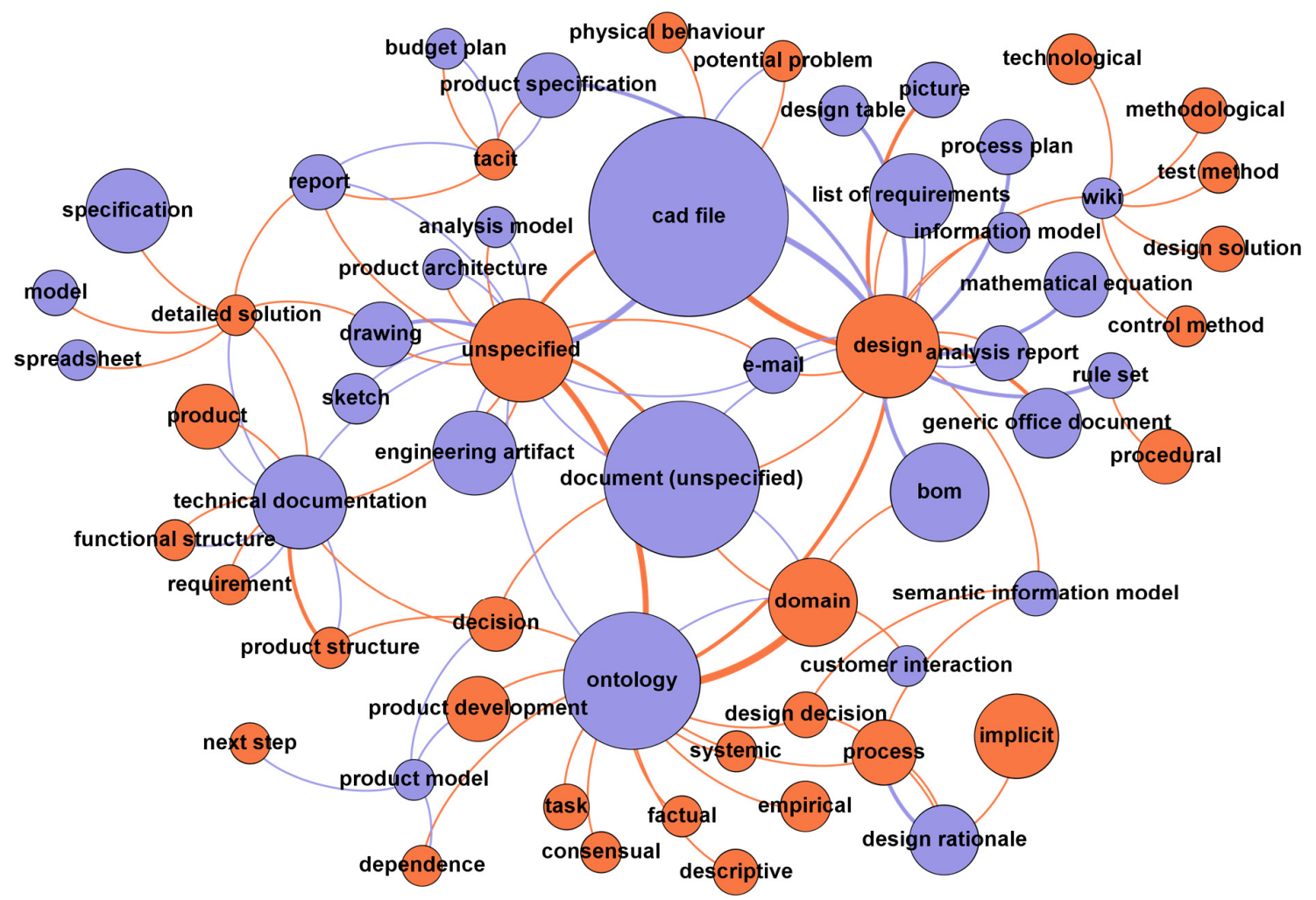

Figure 4. Biggest network of artifacts (purple) and knowledge (orange)

The top five knowledge types by article count were the following: design (22), unspecified (21), detailed solution (7), domain (6) and tacit (6) knowledge. Regarding the linking degree, the top two knowledge types (design and unspecified knowledge) dominated the top five by being linked at least $300 \%$ more than place three (detailed solution). The high linking degree of design knowledge was caused by the research scope, whereas the linking degree of unspecified knowledge had another reason: Many articles discussed knowledge regarding its connection to artifacts, but did not go into detail by differentiating knowledge types or describing what exactly the researcher means by writing about knowledge. This was also related to the finding that nearly $84 \%$ (26 of 31 ) of artifacts present in the biggest network of knowledge and artifacts were connected to the general knowledge terms design, decision, domain and unspecified knowledge. Three artifacts were highly linked to knowledge types: Ontologies, technical documentations and wikis. Thus, these artifacts are flexible regarding the knowledge stored in them and are therefore suitable for a wide range of applications regarding knowledge storage. 


\subsection{Highly connected artifacts and their direct links to IT systems and knowledge}

Within this section, the top four artifacts by linking degree are analyzed regarding their direct links to knowledge and IT systems. The analysis was limited to the top four artifacts because the artifact at place five by linking degree (product specification) had less than half of the linking degree (42\%) of place four (CAD file, absolute linking degree is 14). As seen in Figure 5 (a), unspecified documents linked to the most general knowledge types and directly linked to a wide range of IT systems. The linking degree shared with PDM/PLM systems was especially high in both directions (from and to unspecified documents). Additionally, the bi-directional linking degree to document repositories and knowledge management was higher than average. Three objects were linked one-directional to unspecified documents: Database and CAE systems, as well decision knowledge.
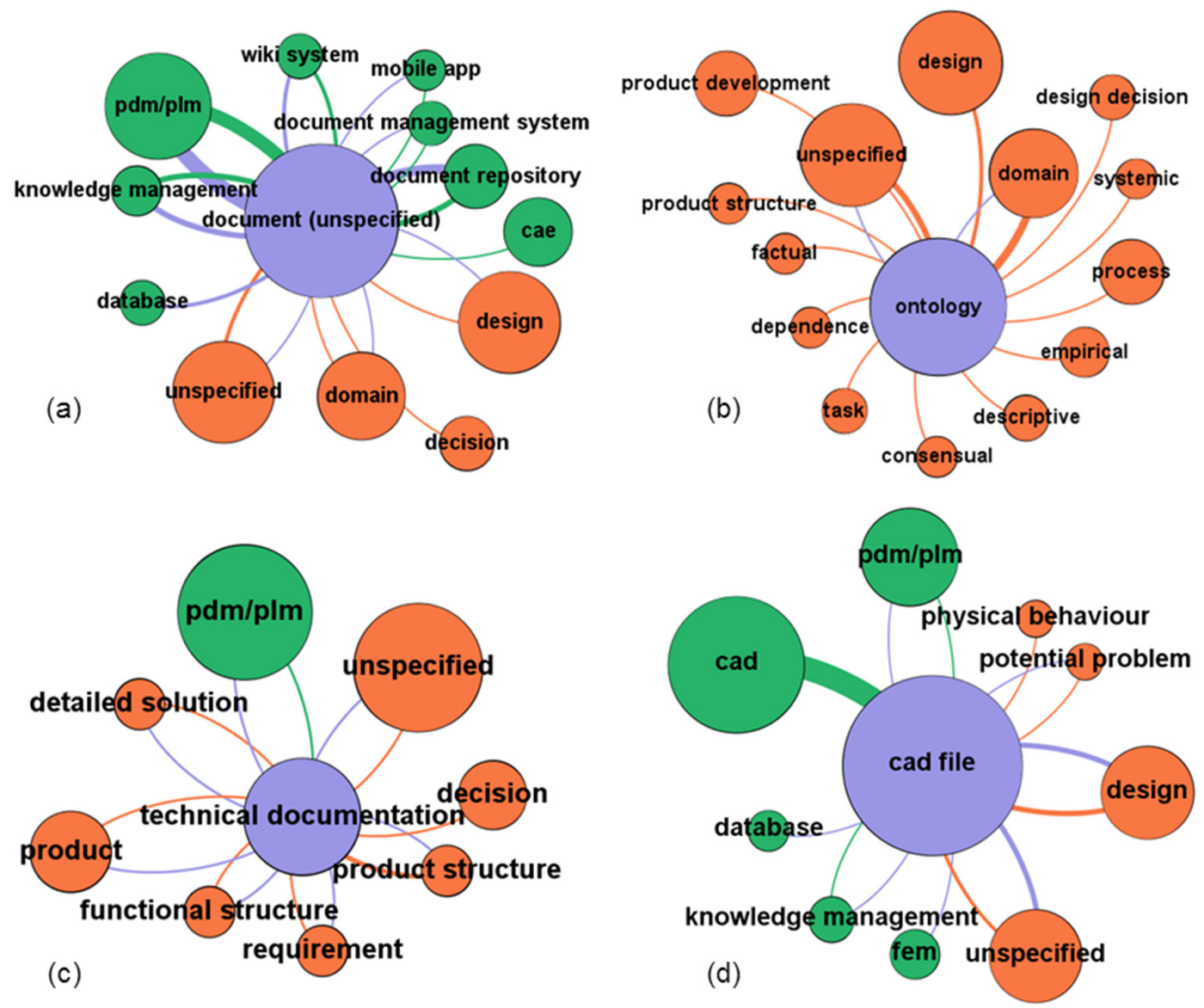

Figure 5. Direct links of highly connected artifacts

As seen in Figure 5 (b), ontologies did not link directly to any IT systems, because ontologies were often used as models to represent relations in research. Therefore, the researcher used ontologies to explain knowledge within engineering, but engineering IT was not mentioned to use ontologies. Regarding the direction of the links it was observed, that ontologies were seen as knowledge storage and not as knowledge source. If ontologies were mentioned as knowledge source, they were the source of general knowledge types: domain, design and unspecified knowledge. As seen in Figure 5 (c), technical documentation were mentioned to be managed within PDM/PLM systems while storing very specific domain knowledge. Additionally, all links were bi-directional, because technical documentation is often used as a communication medium to transfer knowledge between stakeholders. As seen in Figure 5 (d), a wide range of IT systems were mentioned to manage CAD files. CAD systems created CAD files, other systems managed (PDM/PLM, knowledge management), stored (database) and used (FEM) CAD files. CAD files were especially related to the design domain: They stored and provided knowledge 
about the design and potential problems. Knowledge about the physical behavior was stored and a considerable amount of unspecified knowledge was derived from and stored in CAD files. It was expected to find out that $\mathrm{CAD}$ files would also provide knowledge about the physical behavior, but this relation was not found in literature.

\section{Conclusion and outlook}

This article presents the results of a systematic literature review that identified 28 IT systems, 126 artifacts, 78 knowledge types, 103 links between artifacts and IT systems as well as 61 links between artifacts and knowledge types. Additionally, the analysis of relations describes interrelations of these objects. So far, the results of the literature review did not allow for qualitative conclusions such as rankings according to the importance of IT systems, artifacts or knowledge types. The frequency of IT systems, artifacts and knowledge types refers to the number of mentions in the reviewed articles. Consequently, the prominence of e.g. "CAD file" as an artifact could be a result of being one of the most used examples in reviewed literature. Hence, further investigations will be necessary to prove whether the prominence of certain terms also correlates to their importance for the engineering design process. For instance, case studies with practitioners as well as analyzing technical documents of IT systems will lead to increased understanding of this correlation. Another interesting aspect pointed out by the results is the great number of mentions of unspecified documents and knowledge linked to PDM/PLM systems. This strengthens the above-mentioned lack of understanding regarding the connection between IT systems, artifacts and knowledge types in engineering design processes. With regard to knowledge, a proper approach will be needed that can handle its multi-facetted nuances and capture its dynamic flow within complex value creation networks.

\section{Acknowledgement}

This research was funded by the German Research Foundation (DFG, Grant Agreement no. STA 1112/15-1).

\section{References}

Chandrasegaran, S.K., Ramani, K., Sriram, R.D., Horvath, I., Bernard, A. et al. (2013), "The evolution, challenges, and future of knowledge representation in product design systems", Computer-Aided Design, Vol. 45 No. 2 , pp. 204-228. https://doi.org/10.1016/j.cad.2012.08.006

Lehner, F. (2012), Wissensmanagement: Grundlagen, Methoden und technische Unterstützung, 4th ed., Hanser, München. https://doi.org/10.3139/9783446431317

Leonardi, P.M. and Bailey, D.E. (2008), "Transformational technologies and the creation of new work practices: Making implicit knowledge explicit in task-based offshoring”, Mis Quarterly, Vol. 32 No. 2, pp. 411-436. https://doi.org/10.2307/25148846

Lugger, K.-M. and Kraus, H. (2001), "Mastering the human barriers in knowledge management", Journal of Universal Computer Science, Vol. 7 No. 6, pp. 488-497.

Neumann, F. (2015), Analyzing and Modeling Interdisciplinary Product Development, Springer Fachmedien Wiesbaden, Wiesbaden. https://doi.org/10.1007/978-3-658-11092-5

North, K. (2011), Wissensorientierte Unternehmensführung: Wertschöpfung durch Wissen, 5th ed., Gabler Verlag / Springer Fachmedien Wiesbaden GmbH, Wiesbaden. https://doi.org/10.1007/978-3-8349-6427-4

Petersen, K., Feldt, R., Mujtaba, S. and Mattsson, M. (2008), "Systematic Mapping Studies in Software Engineering", Proceedings of the 12th International Conference on Evaluation and Assessment in Software Engineering, BCS Learning \& Development Ltd, Swindon, UK, pp. 68-77.

Snowden, D. (2000), "Organic knowledge management: Part I The ASHEN model: An enabler of action", Knowledge Management, Vol. 3 No. 7, pp. 14-17.

Ullman, D.G. (2010), The mechanical design process, 4th ed., McGraw-Hill Series in Mechanical Engineering, McGraw-Hill, Boston.

Wiater, W. (2007), Wissensmanagement: Eine Einführung für Pädagogen, 1st ed., VS Verlag für Sozialwissenschaften | GWV Fachverlage GmbH Wiesbaden, Wiesbaden.

Maurice Preidel, Research Assistant

Technische Universität Berlin, Industrial Information Technology

Pascalstraße 8-9, 10587 Berlin, Germany

Email: maurice.preidel@tu-berlin.de 